Research Article

\title{
Simulation and Experimental Study of Terahertz Wave Transmission Characteristics Based on Periodic Metal Open Resonant Ring Structures
}

\author{
Yunpeng Ren ${ }^{D}$, ${ }^{1}$ Zhiyu Li, ${ }^{1}$ Yan Chen, ${ }^{2}$ Yunxia Ye, ${ }^{1}$ Zhiduo Xin, ${ }^{1}$ Heng Lu, ${ }^{1}$ Hanyu Wan, \\ and Jianquan Yao ${ }^{1,3}$ \\ ${ }^{1}$ School of Mechanical Engineering, Jiangsu University, Zhenjiang 212013, China \\ ${ }^{2}$ School of Materials Science and Engineering, Jiangsu University, Zhenjiang 212013, China \\ ${ }^{3}$ School of Precision Instrument and Opto-electronics Engineering, Tianjin University, Tianjin 300072, China \\ Correspondence should be addressed to Yunpeng Ren; renyp@ujs.edu.cn
}

Received 3 December 2020; Revised 20 April 2021; Accepted 10 May 2021; Published 17 May 2021

Academic Editor: Sulaiman W. Harun

Copyright (C) 2021 Yunpeng Ren et al. This is an open access article distributed under the Creative Commons Attribution License, which permits unrestricted use, distribution, and reproduction in any medium, provided the original work is properly cited.

Different open resonant ring structures with substrate of polyimide were designed. The transmission characteristics of the structures for terahertz wave were investigated by simulation and experiment. The results show that the transmission peak of the structures moves to high frequency with increase of thickness of the metal layer. With increase of substrate thickness, the transmission peak moved to low frequency and the transmissivity decreased. The influence of number of " $\mathrm{C}$ " shape open resonant rings in the unit structure on the transmission characteristics of terahertz wave was also studied. It is found that when the number of "C" shape open resonant rings increases from one to two, more transmission peaks appeared in the frequency of $0.2-2 \mathrm{THz}$. The transmissivity of the designed structures was tested by terahertz time-domain spectrometer (THz-TDS). The experimental results showed good agreement to the simulation results.

\section{Introduction}

Terahertz $(\mathrm{THz})$ waves are electromagnetic waves with frequencies between $0.1 \mathrm{THz}$ and $10 \mathrm{THz}$ (wavelength between $30 \mu \mathrm{m}$ and $3 \mathrm{~mm}$ ) $[1,2]$. Terahertz wave is located in the transition region from radio wave to infrared light in the electromagnetic spectrum [3]. It is the last band in the electromagnetic spectrum which has not been fully recognized and utilized. The basic characteristics of terahertz wave are high transmissivity, low energy, and fingerprint spectrum. In recent years, due to the development of ultrafast optoelectronic technology, various works have been carried out due to important values of terahertz waves [4-6]. With the rapid development of terahertz technology, a variety of terahertz instruments have been widely applied in medicine, military, aviation, and other fields [7-10], so the demand for high performance terahertz devices is more and more urgent.
Metamaterials and metasurfaces are effective to make modulations to terahertz waves and have drawn intensive interests of investigators for years. The terahertz metamaterial generally consists of periodic metal open resonant ring structure arrays, which have a resonant electromagnetic response $[11,12]$. With this characteristic, specific terahertz wave sensors can be designed. In 1998, Ebbesen found in experiments that when a beam of light is vertically incident to a subwavelength metal hole array structure, the transmission enhancement appears in a specific wavelength range, which is contrary to the traditional classical theory of optical surgery [13]. Since then, researchers have conducted a series of simulations and experiments to modulate electromagnetic waves by using different designs, including terahertz waves. It is found that the dielectric constant of the subwavelength metal structure has a great influence on the resonant transmission frequency [14]. Sun et al. measured the refractive index of $200 \mu \mathrm{m}$ thick copper film and $500 \mu \mathrm{m}$ 
thick aluminum film on silicon substrate by using the terahertz time-domain reflection system and found that the refractive index decreased with increase of $\mathrm{THz}$ frequency [15]. Ma et al. studied the transmission property of terahertz wave and dielectric response of $\mathrm{ZnO}$ conductive film by using $\mathrm{THz}$ time-domain spectrometer (THz-TDS) and obtained the frequency-related conductivity curve and refractive index curve of the film [16]. Carretero-Palacios et al. revealed the influence of thickness of metal films and dielectric constants of substrates on the transmission characteristics of porous metal mesh structures [17]. Currently, most research studies on the transmission characteristics of terahertz wave focus on the influence of different substrates on the transmission characteristics of periodic metal mesh structures [18] and the influence of the thickness of metal film on the transmission characteristics of array structures $[19,20]$. However, it is found that the transmissivity of periodic metal open resonant structures still needs further improvement.

At present, most research studies focus on the realization and regulation of high transmissivity in a single frequency band which limits the applications of terahertz devices. Yen et al. designed a monolayer open resonant ring array and realized the electromagnetic resonance response. The electromagnetic resonance effect of the split-ring resonator (SRR) array was called magnetic plasmon resonance [21]. Xia et al. realized the dual frequency transmission enhancement by employing metal ring arrays [22]. There are majority works that study broadband transmissions of metasurfaces. Compared with visible light, the analysis results of wide terahertz band have important reference significance for the design and preparation of surface plasma devices, such as filters, polarizers and micronano devices.

In this study, different open resonant ring structures based on polyimide substrates are designed. The influence of the structure parameters on the transmission characteristics of the array structure was studied by simulation. The optimal structure parameters obtained from the simulation were employed to fabricate the structures. The experimental results were in good agreement with the simulation results.

\section{Simulation Models}

The different open resonant ring structures were designed as shown in Figure 1. The yellow area is filled with metal, and the rest is polyimide. Figures $1(\mathrm{a}), 1(\mathrm{~b})$, and $1(\mathrm{~d})$ are top view of the structures and Figures 1(c) and 1(e) are side view of corresponding structures. The coordinate system for simulation is also set as in Figure 1. The thickness of the metal layer and the substrate is $t$ and $d$, respectively. The other parameters of the structures can be found in Figures 1(a), $1(b)$, and 1(d). Copper was chosen as the metal layer because it has relatively stable chemical properties and low cost, and polyimide was chosen as the substrate.

\section{Simulation Results and Discussion}

3.1. Numerical Simulation. The numerical simulation was completed by using commercial software CST 2018 based on the time-domain finite integration method. The unit structure models were established in the CST software as shown in Figure 2. The terahertz wave frequency range was set as $0.2-2 \mathrm{THz}$, which is convenient to compare the results from experiments. The background condition was selected as vacuum. The $X$-axis is along the direction of magnetic field or electric field, the $Y$-axis is along the direction of electric field or magnetic field, and the $Z$-axis is along the incident direction of terahertz wave, which is vertical to the unit structure surface. Periodic boundary conditions are set to make the element extend into an infinite plane with the same period in $X$ and $Y$ directions. The terahertz signal serves as the excitation source.

3.2. Results and Discussion. The influence of metal layer thickness on the transmission characteristics of terahertz wave was first studied. The thickness $t$ is set as from $0.2 \mu \mathrm{m}$ to $8 \mu \mathrm{m}$, and the thickness $d$ of substrate is set as $d=10 \mu \mathrm{m}$. The simulation results are shown in Figure 3.

As shown in Figures 3(a), 3(b), and 3(c), with increase of metal thickness, the position of transmission peak of three structures moved to high frequency gradually and the transmissivity slightly decreased. The specific information of transmission peaks for different metal thickness is given in Table 1.

As given in Table 1, it can be found that when the metal thickness is increased from $0.2 \mu \mathrm{m}$ to $8 \mu \mathrm{m}$, the movement of transmission peak is only $7 \mathrm{GHz}, 19 \mathrm{GHz}$, and $9 \mathrm{GHz}$, respectively, for three structures. The transmissivity only decreases $0.4 \%, 0.11 \%$, and $0.11 \%$, respectively. This is because when the metal thickness is bigger than the skin depth, the resonance enhancement will be saturated according to R. Singh's study [23]. The skin depth can be calculated from following equation $[23,24]$ :

$$
\delta=\frac{1}{\sqrt{\pi f \mu_{0} \sigma_{d c}}},
$$

where $f$ is the frequency at which the skin depth is defined, $\mu_{0}$ is the vacuum permeability, and $\sigma_{d c}$ is the DC conductivity of metal. For copper, its skin depth is $85.2 \mathrm{~nm}$ at $0.6 \mathrm{THz}$ which is much smaller than the metal thickness, so the transmission peak moves little when the metal thickness is bigger than $1 \mu \mathrm{m}$.

The influence of substrate thickness on the transmission characteristics was also studied. The substrate thickness of three structures is changed from $5 \mu \mathrm{m}$ to $25 \mu \mathrm{m}$, the metal thickness is selected as $0.2 \mu \mathrm{m}$, and the other parameters keep unchanged. The $12.5 \mu \mathrm{m}$ was selected to compare with experimental results.

The simulation results are shown in Figure 4, and the specific information for the peaks is given in Table 2 .

As given in Table 2, it can be found that for single " $C$ " resonant ring, the transmission peaks shifted to left of 13.2 $\mathrm{GHz}$ from $0.672 \mathrm{THz}$ to $0.540 \mathrm{THz}$ when substrate thickness is increased from $5 \mu \mathrm{m}$ to $25 \mu \mathrm{m}$. The transmissivity is decreased from $99.45 \%$ to $98.94 \%$. For double " $C$ " resonant ring, the transmission peaks shifted to left of 12.8 GHz from $0.643 \mathrm{THz}$ to $0.515 \mathrm{THz}$ when substrate 


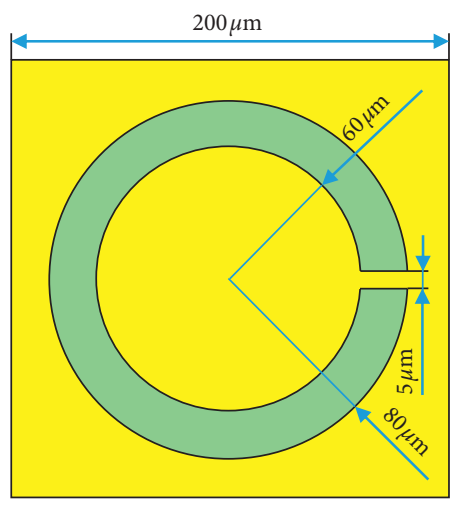

(a)

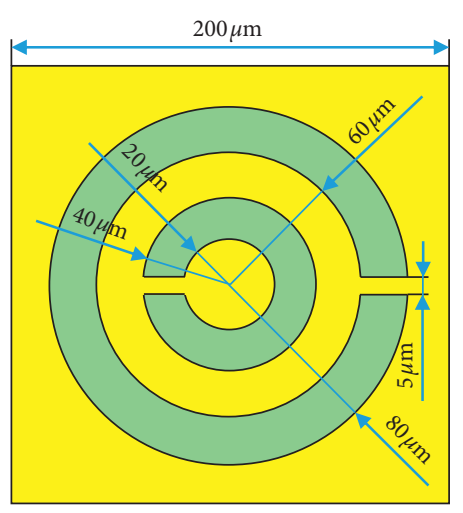

(b)

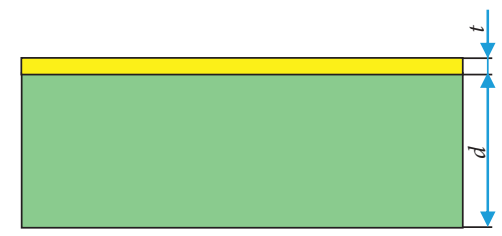

(c)

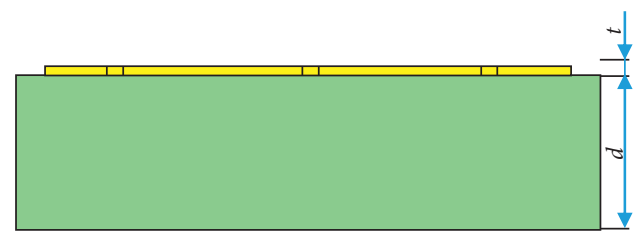

(e)

Figure 1: Schematic and dimension of open resonant ring structures with unit of $\mu \mathrm{m}$. (a) Top view of single "C" resonant ring structure. (b) Top view of double "C" resonant ring structure. (c) Side view of "C" resonant ring structures. (d) Top view of square resonant ring structure. (e) Side view of square resonant ring structure.

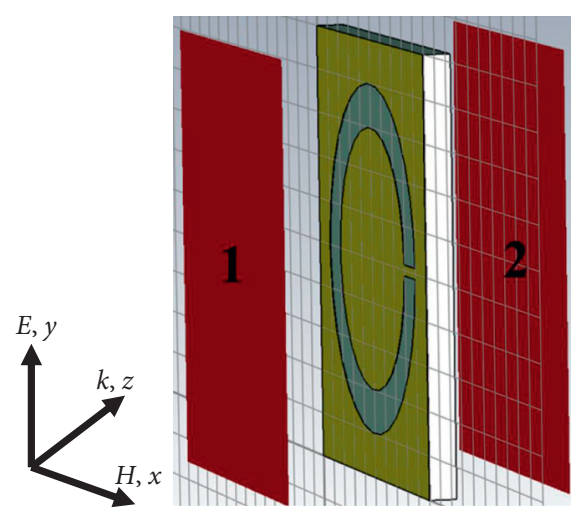

(a)

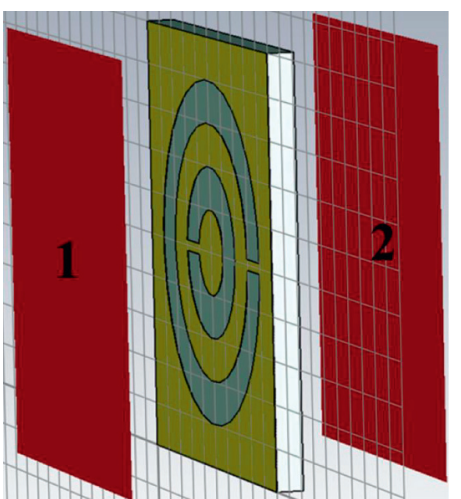

(b)

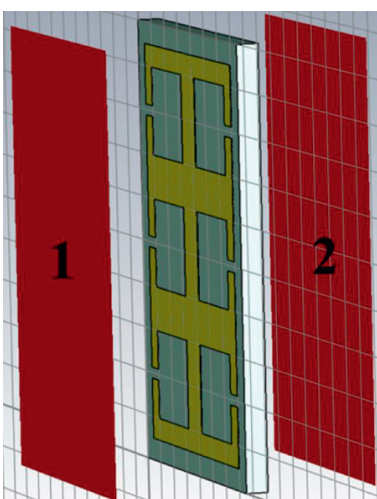

(c)

Figure 2: Schematic of the simulation model. (a) Single "C" resonant ring structure. (b) Double "C" resonant ring structure. (c) Square resonant ring structure.

thickness is increased from $5 \mu \mathrm{m}$ to $25 \mu \mathrm{m}$. The transmissivity is decreased from $98.99 \%$ to $97.34 \%$. For square resonant ring, the transmission peaks shifted to left of $10 \mathrm{GHz}$ from $0.694 \mathrm{THz}$ to $0.594 \mathrm{THz}$ when substrate thickness is increased from $5 \mu \mathrm{m}$ to $25 \mu \mathrm{m}$. The transmission rate is decreased from $99.51 \%$ to $98.82 \%$.

It is shown that when a terahertz wave is perpendicularly incident to a specific surface structure, the excitation 


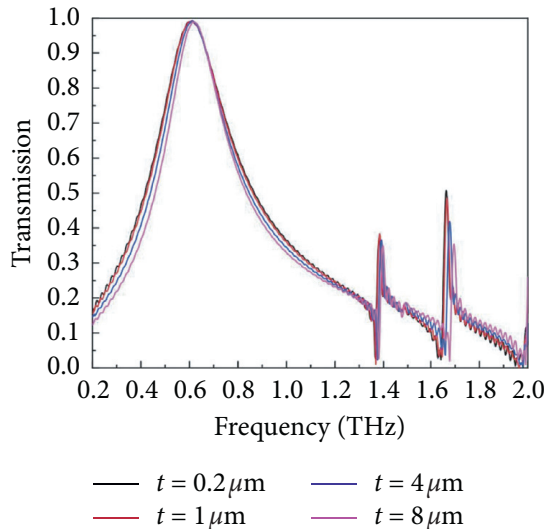

(a)
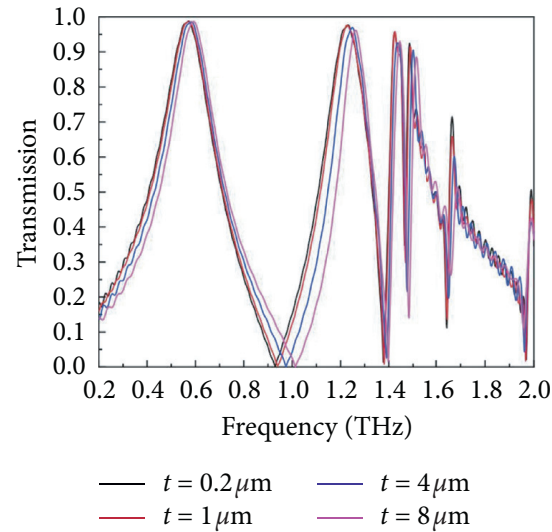

(b)

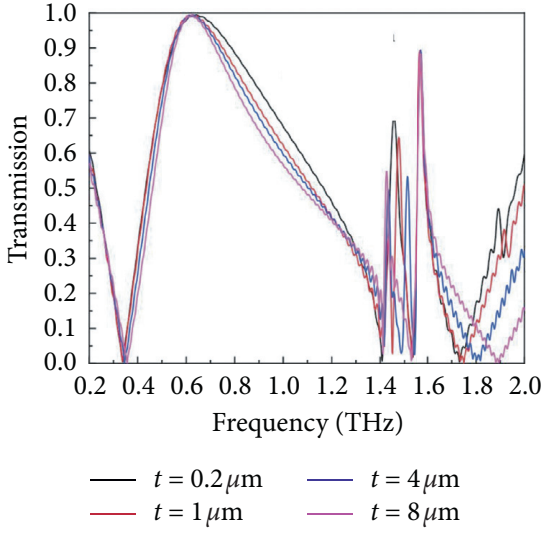

(c)

Figure 3: Transmission spectrum for different thickness of metal layer. (a) Single "C" resonant ring structure. (b) Double "C" resonant ring structure. (c) Square resonant ring structure.

TABLE 1: Transmission peak of resonant ring with different metal layer thickness.

\begin{tabular}{lccc}
\hline Structure & Thickness of metal layer $(\mu \mathrm{m})$ & Transmission peak frequency $(\mathrm{THz})$ & Transmissivity $(\%)$ \\
\hline \multirow{3}{*}{ Single “C" resonant ring } & 0.2 & 0.610 & 99.33 \\
& 1 & 0.614 & 99.21 \\
& 4 & 0.615 & 99.16 \\
& 8 & 0.617 & 98.92 \\
Double "C" resonant ring & 0.2 & 0.573 & 98.78 \\
& 1 & 0.574 & 98.61 \\
& 4 & 0.583 & 98.58 \\
& 8 & 0.592 & 98.67 \\
Square resonant ring & 0.2 & 0.623 & 99.43 \\
& 1 & 0.625 & 99.45 \\
& 4 & 0.628 & 99.39 \\
\end{tabular}
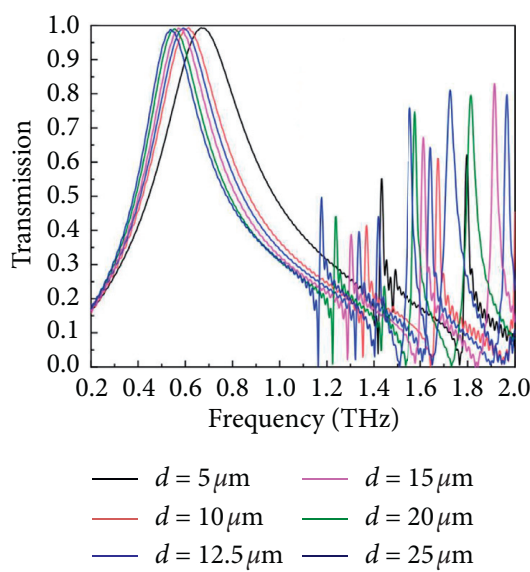

(a)
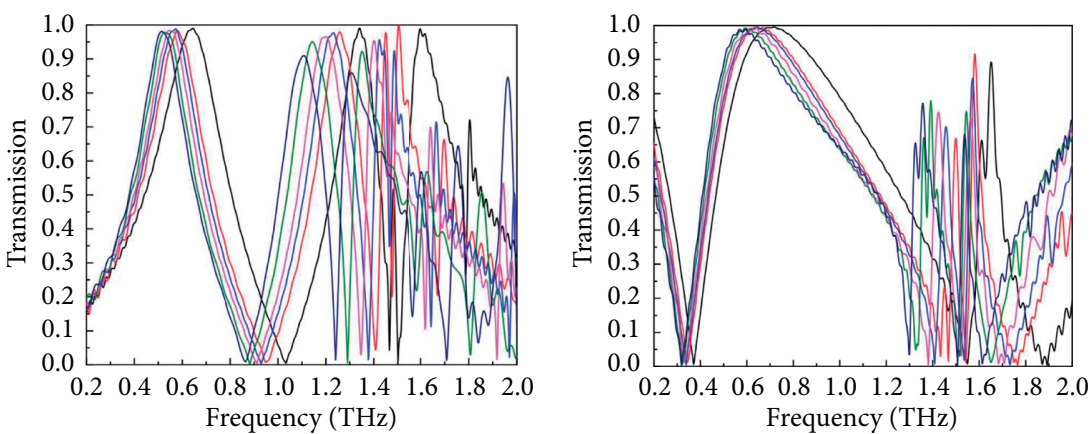

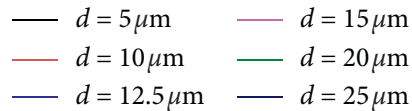

(b)

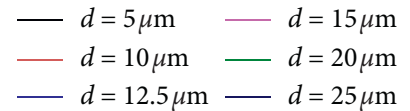

(c)

Figure 4: Transmission spectrum for different thickness of substrate. (a) Single "C" resonant ring structure. (b) Double "C" resonant ring structure. (c) Square resonant ring structure. 
TABLE 2: Transmission of resonant ring with different substrate thickness.

\begin{tabular}{lccc}
\hline Structure & Thickness of substrate $(\mu \mathrm{m})$ & Transmission peak frequency $(\mathrm{THz})$ & Transmissivity $(\%)$ \\
\hline & 5 & 0.672 & 9.610 \\
Single "C" resonant ring & 10 & 0.592 & 99.33 \\
& 12.5 & 0.573 & 99.29 \\
& 15 & 0.555 & 99.25 \\
& 20 & 0.540 & 99.06 \\
& 25 & 0.643 & 98.94 \\
\hline & 5 & 0.573 & 98.99 \\
Double "C" resonant ring & 10 & 0.567 & 98.92 \\
& 12.5 & 0.544 & 98.88 \\
& 15 & 0.529 & 98.40 \\
& 20 & 0.515 & 97.82 \\
Square resonant ring & 25 & 0.694 & 97.34 \\
& 5 & 0.668 & 99.51 \\
& 10 & 0.643 & 99.37 \\
& 12.5 & 0.636 & 99.43 \\
& 15 & 0.614 & 99.55 \\
& 20 & 0.594 & 99.22 \\
\hline
\end{tabular}

wavelength of plasmon resonance in the metal array with holes is approximate [25].

$$
\lambda_{S P}=\frac{L}{\sqrt{m^{2}+k^{2}}} \sqrt{\frac{\varepsilon_{1} \varepsilon_{2}}{\varepsilon_{1}+\varepsilon_{2}}} .
$$

In this formula, $\varepsilon_{1}$ represents the effective dielectric constant of the surrounding medium, $L$ is the lattice constant, $m$ and $k$ represent the different modes of light, and their values are integers. $\varepsilon_{2}=\varepsilon_{r 2}+\varepsilon_{i 2}$ is the dielectric constant of the metal, and $\varepsilon_{r 2}$ and $\varepsilon_{i 2}$ represent the real part and the imaginary part, respectively. The real and imaginary parts are related to the frequency of incident light, the period and size of the hole, the material of the metal, and other factors. In our actual application, the thickness of the substrate of a metal array structure is much smaller than $(c / \omega)$ with $c$ of light speed and $\omega$ of frequency of incident light. So, the structures including substrate are generally considered as thin film. For films that are exposed to the air, the refractive index can be calculated by [26]

$$
n(\omega)=\sqrt{2 A(\omega)-1}
$$

In this formula, $A(\omega)=(c / \omega d)\left|\left(E_{\text {film }} / E_{\text {ref }}\right)-1\right| . E_{\text {film }}$ and $E_{\text {ref }}$ represent the electric field after the light wave penetrates the medium thin film and the reference electric field when the light wave propagates in the free space. $\left(E_{\text {film }} / E_{r e f}\right)$ represents the transmittance of light waves through a dielectric film, and this value is related to the thickness of the substrate film of the structure. In addition to the resonant transmission factors, the frequency selection effect of the subwavelength metal array with holes on the surface of terahertz wave will also change the transmission properties of the substrates of the structure. Considering $\varepsilon(\omega)=n^{2}(\omega)$, which is the relation between transmittance and dielectric constant of substrate material, we can conclude that

$$
\varepsilon_{1}(\omega)=2 \frac{c}{\omega d}\left|\frac{E_{\text {film }}}{E_{\text {ref }}}-1\right|-1
$$

According to equation (4), the terahertz wave transmittance of the subwavelength metal array structure is directly related to the dielectric constant of the substrate as well as the thickness of the substrate as a whole, and changes in the thickness of the substrate $d$ also leads to changes in $\varepsilon_{1}$. According to equation (2), we can find that the change of $\varepsilon_{1}$ will cause the shift of subwavelength metal array structure of surface plasmon resonance wavelength. Numerical simulation for equations (2)-(4) is carried out to verify the reasoning, and the results are shown in Figure 5. It can be found that the transmission peak position of the metal hole array structure is subject to change with the change of substrate thickness $d$. When the thickness of the substrate increases, the position of the transmission peak moves to low frequency. Substrate thickness has great influence to the dielectric constant of metal microstructure. Due to this, the surface plasmon resonance wavelength will be changed and lead to the movement of the position of transmission peak.

Figure 5 shows the relationship between the frequency of transmission peak and the thickness of the substrate. It can be found that the frequency of transmission peak decreases nonlinearly. With increase of thickness of substrate, the frequency of transmission peak tends to be stable. According to the literature [27], the main reason to affect the resonance frequency is the interface plasma resonance between metal and substrate. If the substrate is thick enough, it will be like a half infinite medium, and the interface effect will have nothing to do with the transmission peak frequency. Due to this reason, the transmission peak frequency will tend to a stable position. In practical application, we can realize the modulation of terahertz wave by changing thickness of substrate in a certain range.

In order to better understand the transmission characteristics of the three proposed structures, the electric energy density of the unit structures at their respective resonant frequencies were studied and are shown in Figure 6. As 


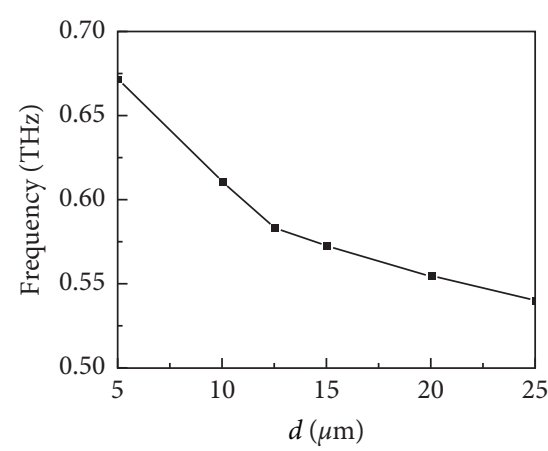

(a)

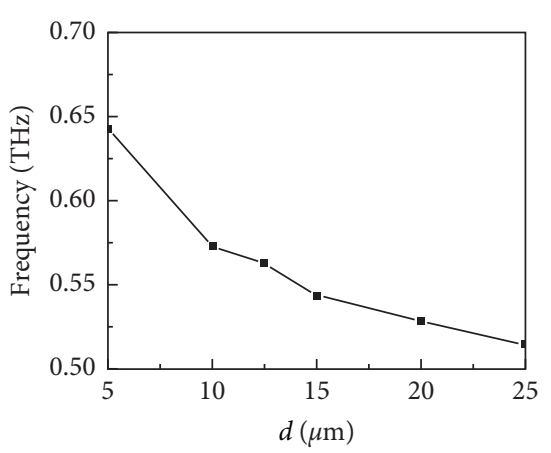

(b)

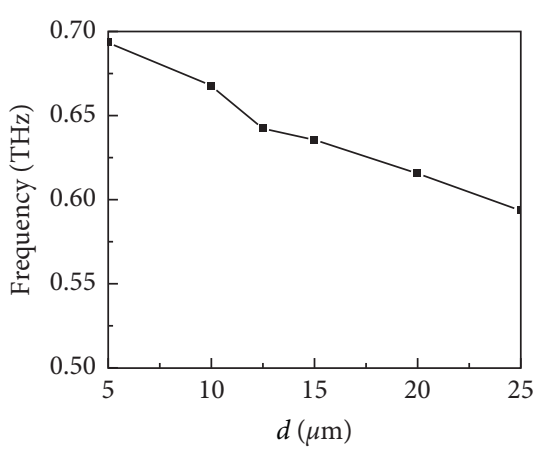

(c)

FIGURE 5: Relationship between frequency of peak transmission and substrate thickness. (a) Single "C" resonant ring structure. (b) Double "C" resonant ring structure. (c) Square resonant ring structure.

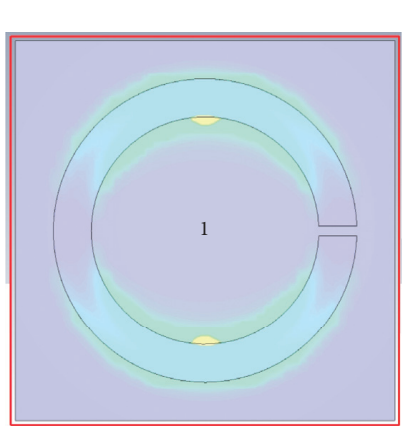

(a)
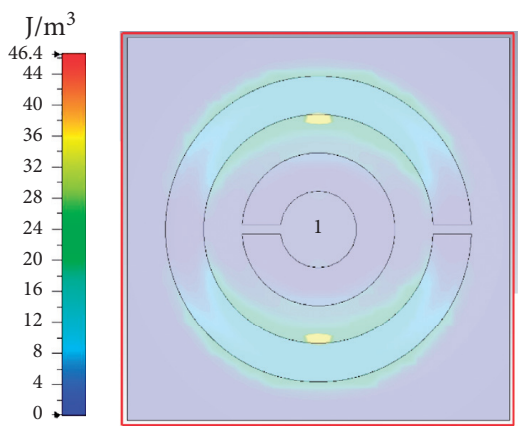

(b)
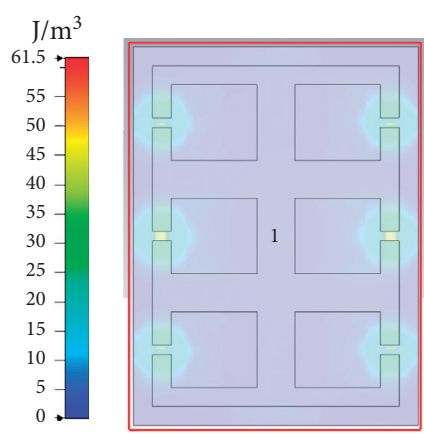

(c)

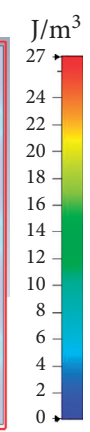

Figure 6: Electric energy density of the three structures. (a) Single " $\mathrm{C}$ " resonant ring structure $\left((\mathrm{f})_{1}=0.592 \mathrm{THz}\right)$. (b) Double " $\mathrm{C}$ ” resonant ring structure $\left(f_{2}=0.592 \mathrm{THz}\right)$. (c) Square resonant ring structure $\left(f_{3}=0.592 \mathrm{THz}\right)$.

shown in Figures 6(a) and 6(b), since the ring is hollow and the opening is metal, the energy of the incident terahertz wave is concentrated on both sides of the ring, revealing a stronger field confinement and $\mathrm{THz}$ wave capture [28, 29]. As shown in Figure 6(c), the electric field intensity is evenly and symmetrically distributed among the six openings, and this distribution is the formation of electric field dipole in the ring. Also, the energy density is not very high. This is because the opposite flow of the induced cycle on the surface cancels out the creation of magnetic dipoles.

The influence of the number of rings on transmission characteristics was studied. Single and double "C" resonant ring were selected for comparison. The metal layer thickness was $0.2 \mu \mathrm{m}$, and the substrate thickness was $12.5 \mu \mathrm{m}$.

As shown in Figure 7, for single "C" resonant ring structure, only one high transmission peak appears at $0.592 \mathrm{THz}$ with transmissivity of $99.29 \%$. The bandwidth with transmissivity above $90 \%$ is $0.117 \mathrm{THz}$. For double "C" resonant ring structure, four high transmission peaks of F1, F2, F3, and F4 appear at $0.567 \mathrm{THz}, 1.218 \mathrm{THz}$, $1.421 \mathrm{THz}$, and $1.487 \mathrm{THz}$, respectively. For peaks F1 and F2, the bandwidth with transmissivity above $90 \%$ is $86 \mathrm{GHz}$ and $72 \mathrm{GHz}$, respectively. For peaks F3 and F4, the corresponding bandwidth is too narrow to discuss. The detailed information for above simulation results is given in Table 3.

\section{Experimental Results and Discussion}

4.1. Experiment. According to the optimal parameters obtained from the previous simulation and considering the difficulty of the actual sample processing, copper with a thickness of $0.2 \mu \mathrm{m}$ was selected as the metal layer and polyimide with a thickness of $12.5 \mu \mathrm{m}$ as the substrate for the sample preparation.

Polyimide (PI) with thickness of $12.5 \mu \mathrm{m}$ was used as the substrate for the three structures in the experiment. First, a layer of polyimide with thickness of $12.5 \mu \mathrm{m}$ was coated on the silicon chip with thickness of $500 \mu \mathrm{m}$ by PI spinning. Then, the open resonant ring structures were formed on the PI substrate by positive photolithography through homogenization, predrying, preexposure, overdrying, overexposure, development, and other processes. The AZ5214 model was selected as the photoresist, and $0.2 \mu \mathrm{m}$ thick copper was plated on the PI by magnetron sputtering. After stripping, an open ring copper film was formed on the PI substrate. Disco cutting machine (Disco 641) with a hard knife was used to slice the samples into small pieces of $8 \mathrm{~mm} \times 8 \mathrm{~mm}$. The final samples were obtained after soaking in HF (40\%) for 3 min and separated from the silicon wafers. The microstructures of final samples are shown in Figure 8.

The detection of transmission characteristics of the samples was carried on the THz-TDS system (Advantest, 


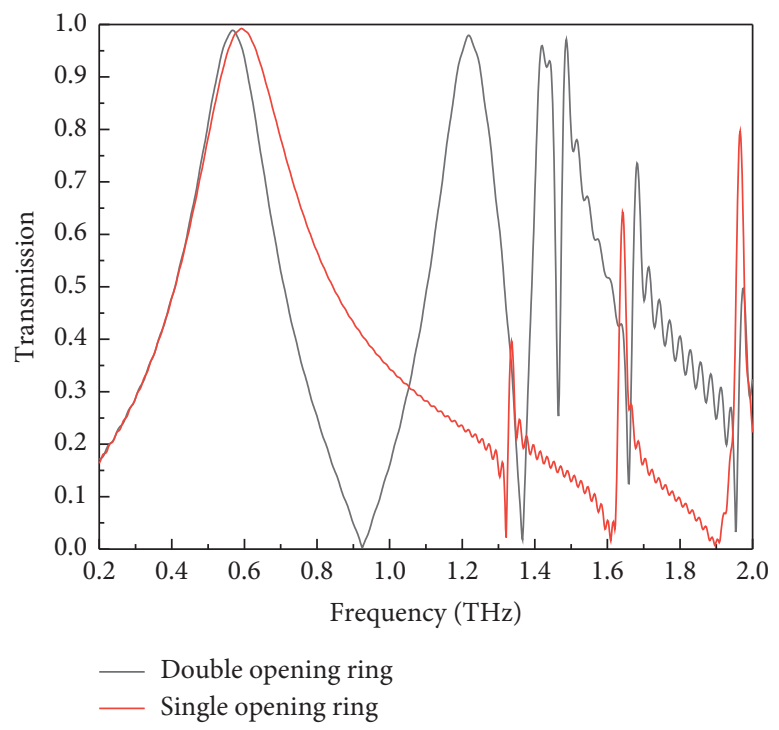

Figure 7: Simulated transmission spectrum for single and double "C" resonant ring structure.

TABLE 3: The simulation of transmission peak of single and double " $\mathrm{C}$ " resonant ring.

\begin{tabular}{lccc}
\hline Structure & Transmission peak & Transmission peak frequency $(\mathrm{THz})$ & Transmissivity $(\%)$ \\
\hline Single “C” resonant ring & F1 & 0.592 & 99.29 \\
& F1 & 0.567 & 98.88 \\
Double “C” resonant ring & F2 & 1.218 & 97.93 \\
& F3 & 1.421 & 95.92 \\
& F4 & 1.487 & 97.13 \\
\hline
\end{tabular}

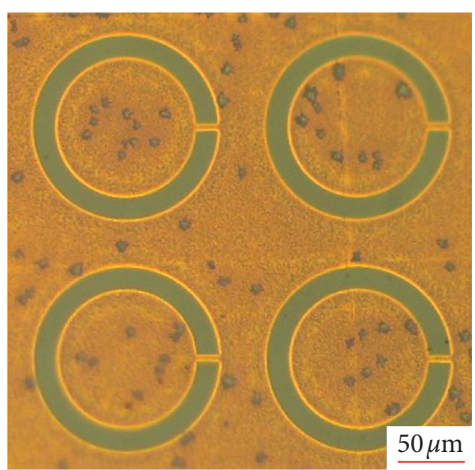

(a)

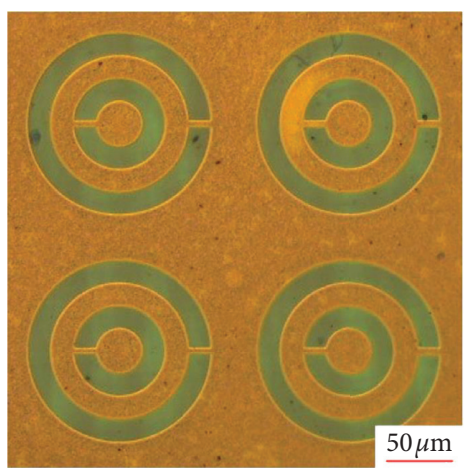

(b)

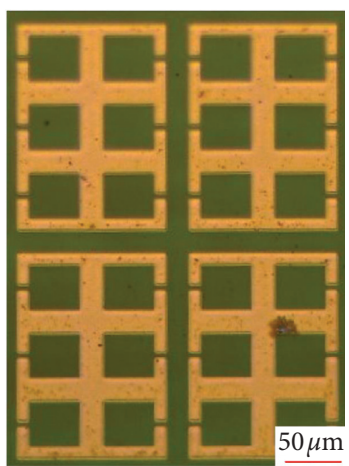

(c)

FIGURE 8: Microstructure of resonant ring structures. (a) Single "C" resonant ring structure. (b) Double "C" resonant ring structure. (c) Square resonant ring structure.

TAS7400). The system is mainly composed by a femtosecond laser, electric control device, and $\mathrm{THz}$ wave generating device. The Ti: sapphire femtosecond laser with pulse width of $100 \mathrm{fs}$, center wavelength of $800 \mathrm{~nm}$, and repetition rate of $82 \mathrm{MHz}$ is used as the light source. The femtosecond laser pulse is divided into two beams. One beam is used to generate terahertz pulse, and the other one acts as a probe beam to detect the terahertz pulse. The detection width of this system is $0.1-5 \mathrm{THz}$. The temperature is kept at $21^{\circ} \mathrm{C}$ during the experiment, and the humidity of the detection environment is controlled below $4 \%$ by the air dryer so as to minimize the absorption of water to terahertz wave and increase the accuracy of the detection results.

The transmissivity can be calculated by following equation:

$$
T(v)=\frac{\text { Power }_{\text {transmitted }}(v)}{\text { Power }_{\text {reference }}(v)},
$$

where $T(\mathrm{v})$ is the transmissivity, $v$ is the incident pulse frequency, Power $_{\text {reference }}(v)$ is the energy of incident pulse, and Power transmitted $(v)$ is the energy of transmission pulse. 


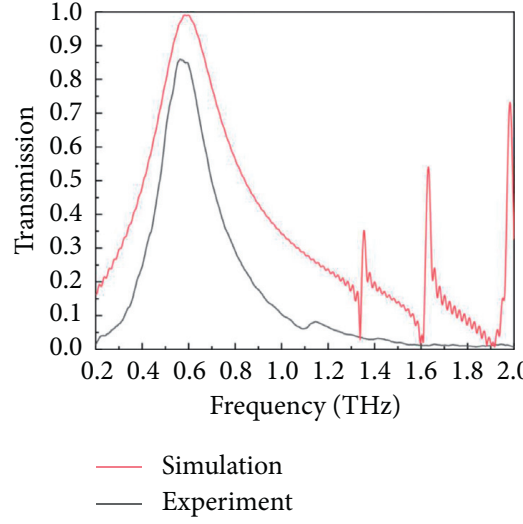

(a)

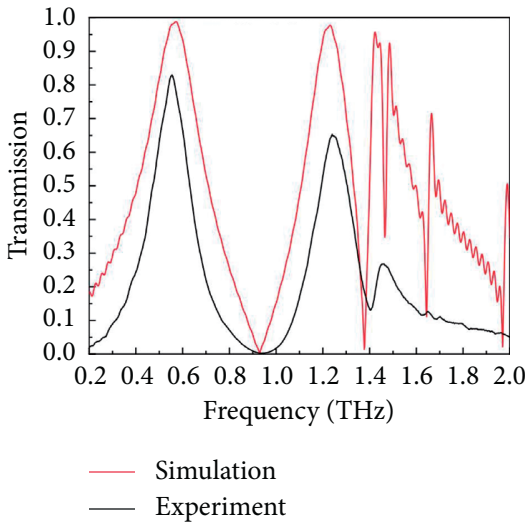

(b)

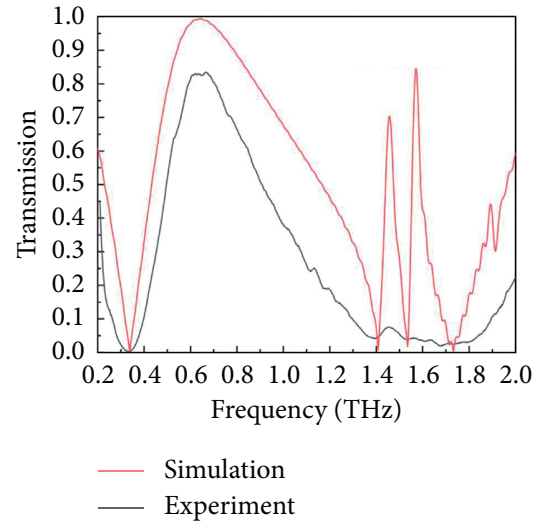

(c)

Figure 9: Comparison simulation and experimental results of transmission spectrum for three structures. (a) Single "C" resonant ring structure. (b) Double "C" resonant ring structure. (c) Square resonant ring structure.

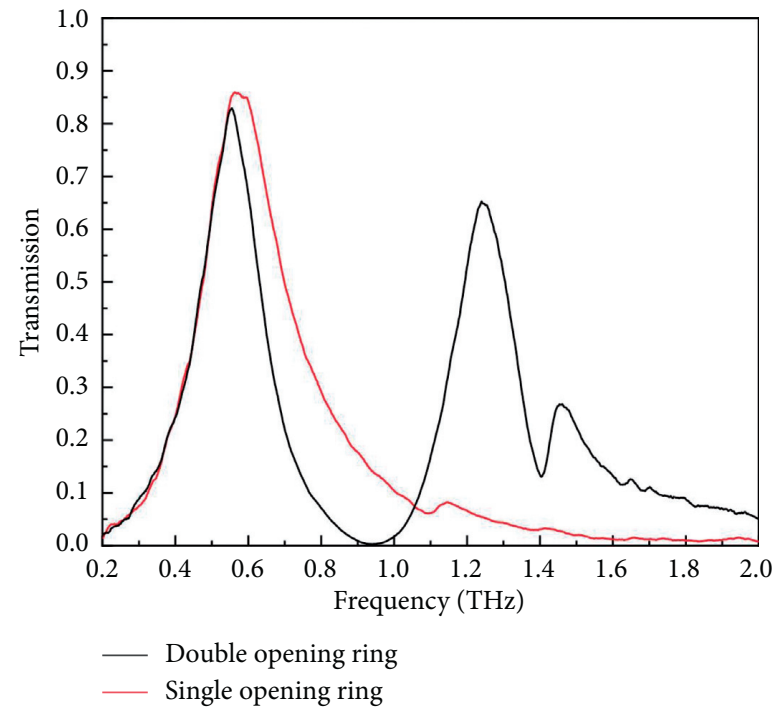

FIgURE 10: Experimental transmission spectrum for single and double "C" resonant ring structures.

4.2. Results and Discussion. As shown in Figure 9(a), the transmission peak of the sample was at $0.56 \mathrm{THz}$ with transmissivity of $85.9 \%$ for single " $\mathrm{C}$ " resonant structure. For double " $\mathrm{C}$ " resonant structure, the transmission peak showed up at $0.56 \mathrm{THz}$ with transmissivity of $82.9 \%$, as shown in Figure 9(b). As for the square resonant ring structure, the transmission peak was at $0.62 \mathrm{THz}$ with transmissivity of $84.1 \%$. As shown in Figure 9, it can be found that the transmission peak position has a very good match for the simulation and the experimental results. The difference of transmissivity is mainly due to the absorption of polyimide substrate for $\mathrm{THz}$ wave and the slight oxidation of the metal layer. Also, there are more transmission peaks in simulation than in experiment. The main reason is due to the absorption of polyimide substrate for $\mathrm{THz}$ wave and the slight oxidation of the metal layer. Besides, the size error of the samples and the absorption of terahertz wave by water in the environment during detection process will also lead to a lower transmission compared to the ideal simulation results.

Figure 10 shows the comparison of transmission spectrum for single and double " $\mathrm{C}$ " resonant ring structures. Table 4 provides specific information for the peaks of experiment result. It can be seen from the diagram for the single " $\mathrm{C}$ " resonant ring structure, only one supertransmission peak appears within $0.2-2 \mathrm{THz}$. However, for the double " $C$ " resonant ring structure, three transmission peaks show up at $0.561 \mathrm{THz}, 1.232 \mathrm{THz}$, and $1.474 \mathrm{THz}$ with transmissivity of $84.2 \%, 66.2 \%$, and $28.1 \%$, respectively. This is showing the potential to improve the sensitivity of $\mathrm{THz}$ detection devices by adding more resonant rings to the unit structure. In order to improve the transmissivity of the structures, other materials with low terahertz absorption 
TABLE 4: The experiment of transmission peak of single and double "C" resonant ring.

\begin{tabular}{lccc}
\hline Structure & Transmission peak & Transmission peak frequency (THz) & Transmissivity $(\%)$ \\
\hline Single "C" resonant ring & F1 & 0.592 & 83.4 \\
& F1 & 0.561 & 84.2 \\
Double "C" resonant ring & F2 & 1.232 & 66.2 \\
& F3 & 1.474 & 28.1 \\
\hline
\end{tabular}

such as polyethylene [30] or low-density polyethylene [31] can be considered in the future study.

\section{Conclusions}

Three kinds of open ring structures were designed, and their transmission characteristics of $\mathrm{THz}$ waves were studied by simulation and experiments. The influence of metal layer thickness, substrate thickness, and number of open rings on the transmission characteristics was studied.

The results show that the transmissivity decreases with increase of thickness of metal layer and substrate. The transmission peaks have a redshift with increase of thickness of metal layer and substrate, and the influence from substrate is greater than that from metal layer. For single " $\mathrm{C}$ " and square open resonance ring structures, only one resonant transmission peak with transmissivity higher than $90 \%$ appears in the range of $0.2-2 \mathrm{THz}$. However, for double " $\mathrm{C}$ " open resonant ring structure, four transmission peaks with transmissivity higher than $90 \%$ appear in the range of $0.2-2 \mathrm{THz}$. According to the actual demand, by changing the thickness of the metal layer, as well as the thickness of the substrate, adjust the terahertz wave transmission and its transmission peak position, so as to achieve the desired purpose.

The transmission characteristics of three structures were studied experimentally. The results of position of transmission peaks have a good agreement with simulation. The transmissivity of experiments is lower than the simulation results which is mainly because the simulation was carried out in an ideal environment first, and the size error in the sample preparation process and the absorption of terahertz wave by water in the environment during the detection process were caused.

\section{Data Availability}

The data used to support the findings of this study are included within the article.

\section{Conflicts of Interest}

The authors declare that they have no conflicts of interest.

\section{Acknowledgments}

The authors are grateful to the projects supported by the National Nature Science Foundation of China (11504144), the project funded by the Talent Starting Foundation of Jiangsu University (15JDG133), and the Young Leading Teachers Project of Jiangsu University.

\section{References}

[1] M. Tonouchi, "Cutting-edge terahertz technology," Nature Photonics, vol. 1, no. 2, pp. 97-105, 2007.

[2] Z. H. He, Y. P. Zhang, H. Y. Zhang, Q. M. Zhang, J. H. Liao, and Y. H. Zhou, "Study of optimal cavity parameter in optically pumped $\mathrm{D}_{2} \mathrm{O}$ gas terahertz laser," Journal of Infrared Millimeter and Terahertz Waves, vol. 31, pp. 551-558, 2010.

[3] T. Chen, "Terahertz spectra identification of biomolecules based on principal component analysis and fuzzy recognition," Chinese Journal of Quantum Electronics, vol. 33, pp. 392-398, 2016.

[4] H. Tao, W. J. Padilla, and R. D. Averitt, "Recent progress in electromagnetic metamaterial devices for terahertz applications," IEEE Journal of Selected Topics in Quantum Electronics, vol. 17, no. 1, pp. 92-101, 2011.

[5] L. J. Liang, X. Yan, J. Q. Yao, G. C. Tian, and D. Xue, "Two dimensional left-handed material based on parallel metallic double rods in terahertz wave," Acta Optica Sinica, vol. 32, no. 3, Article ID 0316001, 2012.

[6] Q. Li, Z. W. Xia, R. Yao, S. H. Ding, and Q. Wang, "Analysis of terahertz array imaging system using optical design software," Chinese Journal of Lasers, vol. 38, no. 4, Article ID 0411001, 2011.

[7] G. Gallot, "Terahertz sensing in biology and medicine," Photoniques, vol. 101, no. 101, pp. 53-58, 2020.

[8] K.-D. Xu, J. Li, A. Zhang, and Q. Chen, "Tunable multi-band terahertz absorber using a single-layer square graphene ring structure with T-shaped graphene strips," Optics Express, vol. 28, no. 8, pp. 11482-11492, 2020.

[9] K. D. Xu, Y. Cai, X. Cao, Y. Guo, and Q. Chen, "Multi-band thz absorbers using t-shaped slot-patterned graphene and its complementary structure," Journal of the Optical Society of America B, vol. 37, no. 10, pp. 2034-3040, 2020.

[10] H. Xiong and Q. Shen, "A thermally and electrically dualtunable absorber based on Dirac semimetal and strontium titanate," Nanoscale, vol. 12, no. 27, pp. 14598-14604, 2020.

[11] Y. Ma, Q. Chen, J. Grant, S. C. Saha, A. Khalid, and D. R. S. Cumming, "A terahertz polarization insensitive dual band metamaterial absorber," Optics Letters, vol. 36, no. 6, pp. 945-947, 2011.

[12] L. T. Guo, K. J. Mu, and C. Deng, "Terahertz spectroscopy and imaging," Infrared and Laser Engineering, vol. 42, no. 1, pp. 51-56, 2013.

[13] T. W. Ebbesen, H. J. Lezec, H. F. Ghaemi, T. Thio, P. A. Wolff, and P. A. Wolff, "Extraordinary optical transmission through sub-wavelength hole arrays," Nature, vol. 391, no. 6668, pp. 667-669, 1998.

[14] T. H. Meng, G. Z. Zhao, and C. L. Zhang, "Study of enhanced transmission of terahertz radiation through subwavelength fractals structures," Acta Physical Sinica, vol. 57, pp. 38463852, 2008.

[15] W. F. Sun, X. K. Wang, and Y. Zhang, "Measurement of refractive index for high reflectance materials with terahertz time domain reflection spectroscopy," Chinese Physics Letters, vol. 26, no. 11, pp. 4210-4214, 2009. 
[16] G. H. Ma, D. Li, H. Ma, and J. Shen, "Carrier concentration dependence of terahertz transmission on conducting $\mathrm{ZnO}$ films," Applied Physics Letters, vol. 93, no. 21, pp. 1101-1104, 2008.

[17] S. Carretero-Palacios, F. J. Garcia-Vidal, and L. MartinMoreno, "Effect of film thickness and dielectric environment on optical transmission through subwavelength holes," Physical Review B, vol. 85, no. 03, pp. 5417-5426, 2012.

[18] F. J. Garcia-Vidal, E. Moreno, J. A. Porto, and L. MartinMoreno, "Transmission of light through a single rectangular hole," Physical Review Letters, vol. 95, no. 10, pp. 3901-3905, 2005.

[19] M. T. Reiten, D. Roy Chowdhury, J. Zhou, and A. J. Taylor, "Resonance tuning behavior in closely spaced inhomogeneous bilayer metamaterials," Applied Physics Letters, vol. 98, no. 13, pp. 1105-1108, 2011.

[20] V. G. Bordo, "Model of Fabry-Perot-type electromagnetic modes of a cylindrical nanowire," Physical Review B, vol. 81, no. 3, pp. 5420-5430, 2010.

[21] T. J. Yen, W. J. Padilla, N. Fang et al., "Terahertz magnetic response from artificial materials," Science, vol. 303, no. 5663, pp. 1494-1496, 2004.

[22] S. Xia, D. Yang, T. Li, X. Liu, and J. Wang, "Role of surface plasmon resonant modes in anomalous terahertz transmission through double-layer metal loop arrays," Optics Letters, vol. 39, no. 5, pp. 1270-1273, 2014.

[23] R. Singh, E. Smirnova, A. J. Taylor, J. F. O’Hara, and W. Zhang, "Optically thin terahertz metamaterials," Optics Express, vol. 16, no. 9, pp. 6537-6543, 2008.

[24] S. Ramo and J. R. Whinnery, "Fields and waves in modern radio," Physics Today, vol. 7, no. 10, p. 50, 1954.

[25] Z. P. Jiang, M. Li, and X. C. Zhang, "Dielectric constant measurement of thin films by differential time-domain spectroscopy," Applied Physics Letters, vol. 76, no. 22, 2000.

[26] H. Ghaemi, T. Thio, D. E. Grupp, T. W. Ebbesen, and H. J. Lezec, "Surface plasmons enhance optical transmission through subwavelength holes," Physical Review B, vol. 58, no. $11,1998$.

[27] J. G. Han, X. C. Lu, and W. L. Zhang, "Terahertz transmission in subwavelength holes of asymmetric metal dielectric interfaces: the effect of dielectric layer," Journal of Applied Physics, vol. 103, no. 3, Article ID 033108, 2008.

[28] Y. Cheng, H. Zhao, and C. Li, "Broadband tunable terahertz metasurface absorber based on complementary-wheel-shaped graphene," Optical Materials, vol. 109, no. 1, Article ID 110369, 2020.

[29] H. Zou and Y. Cheng, "Design of a six-band terahertz metamaterial absorber for temperature sensing application," Optical Materials, vol. 88, pp. 674-679, 2019.

[30] Y. Ma, A. Khalid, T. D. Drysdale, and D. R. S. Cumming, "Direct fabrication of terahertz optical devices on low-absorption polymer substrates," Optics Letters, vol. 34, no. 10, pp. 1555-1557, 2009.

[31] S. Y. Benjamin, F. Christophe, L. Hungyen, and A. Derek, "Low-cost ultra-thin broadband terahertz beam-splitter," Optical Express, vol. 20, no. 5, pp. 4968-4978, 2012. 